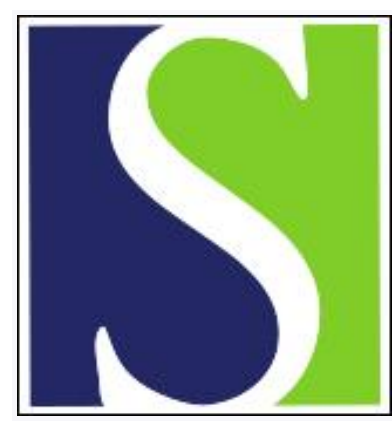

Scand J Work Environ Health 1999;25(6):484-490

https://doi.org/10.5271/sjweh.470

Issue date: Dec 1999

Etiologic research: needed revisions of concepts and principles

by Miettinen OS

Key terms: case-control study; cohort study; confounding; etiologic study; matching

This article in PubMed: www.ncbi.nlm.nih.gov/pubmed/10884143

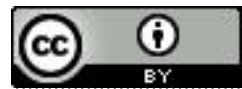




\title{
Etiologic research
}

\section{Needed revisions of concepts and principles}

\author{
by Olli S Miettinen, $M D^{1}$
}

\begin{abstract}
Miettinen OS. Etiologic research: needed revisions of concepts and principles. Scand J Work Environ Health $1999 ; 25$ (6, special issue):484-490.
\end{abstract}

\begin{abstract}
Even though etiologic research has been the central concern in academic epidemiology, its concepts have remained confused or malformed, starting from that of etiology itself; and the same applies to its principles, starting from the notion that the principal variants of an etiologic study are the 'cohort' study and the 'case-control' study. This article suggests revisions of some central concepts pertaining to the object (and objective) of an etiologic study, and it posits an updated conception of the essence - singular - the study itself. This is supplemented by some novel, yet merely orientational, propositions in respect to quality-assurance in etiologic research.
\end{abstract}

Key terms case-control study, cohort study, confounding, etiologic study, matching.

Isaiah Berlin, a leading humanist intellectual of this nowdying 20th century, issues this precept $(1$, p. 61):

... where the concepts are firm, clear and generally accepted and the methods of reasoning and arriving at conclusions are agreed between men (at least the majority of those who have anything to do with these matters), there and only there is it possible to construct a science...

For etiologic research, the core concept naturally is that of etiology itself. The one and only at present available dictionary of epidemiology (2), notably one that is sponsored by the International Epidemiological Association, defines this concepts as follows: "Literally, the science of causes, causality; in common usage, cause." For this to be generally accepted, it would also have to be accepted, for example, that tautology is, literally, the science of unnecessary repetition in other words, verbal redundancy; in common usage, pointless restatement. By the same token, it would have to be accepted that it is not that various sciences involve morphologic - and epidemiologic - issues but that instead, or in addition at least, morphology and epidemiology are, literally, sciences in themselves. No one, I believe, regards morphology as a science while, regrettably, many still take epidemiology to be a science in its own right, literally or otherwise.
As for methods of reasoning and arriving at conclusions about etiology, there now is a stupefying agreement among those concerned that there are three principal options for this: cohort, case-control, and cross-sectional study. At the same time, according to our dictionary (2), cohort is "any designated group of persons followed or traced over a period of time," case is "a person ... having ... the particular disease ... under investigation," control is "person(s) in a comparison group ... that differs from the subjects of the study," and "cross-sectional" designates reference to "one particular time." To agree on this, in its intellectual underpinnings, is analogous to agreeing that there are three ways of arriving at conclusions about the effects of interventions: one defined by population follow-up, another by joint involvement of persons with the outcome of interest and persons who differ from the subjects of the study, and a third by its reference to one particular time. In the 21 st century, I believe, this kind of violation of the imperatives of logic - that or ganon (instrument) of all science since its introduction in antiquity - will come to an end.

I here outline, for etiologic research, some of the central concepts that I expect to become firm, clear and generally accepted in the 21 st century, together with some of the proximal principles of research that flow from these. In so doing, I need to admit, I am revising ideas that I, too, have been seduced to adopt and hold University, 1020 Pine Avenue West, Montreal, Quebec H3A 1A2, Canada. [E-mail: osm@epid.lan.mcgill.ca] 
for much of my four decades of association with this line of research, recently expanded to interest in quintessentially applied medical research at large.

\section{Etiology; etiognosis; etiologic time, determinant and contrast}

Etiology, it seems to me, is a concept rather peculiar to medicine; but regardless, it is from the vantage of medicine that the concept is to be grasped, for the purposes of etiologic research in the health fields.

In medicine, as a case of a particular illness (disease, defect, injury ...) has been securely diagnosed, interest may turn to the question of how it might have come about. In this, the concern tends not to be that of pathogenesis, that is, the sequence of tissue changes resulting in the anomaly that is definitional to the illness. Rather, the focus tends to be on the causal influences that initiated and/or sustained the pathogenetic process, on etiogenesis in this sense (to use a neologism here). It is this causal origin of an illness, of a particular case of it or the illness at large, that constitutes its etiology. Each case of the illness has an etiology, completely unknown perhaps. Whereas knowledge (probabilistic) about the presence of the illness is termed diagnosis, knowledge about its etiology might be termed etiognosis (3).

In any contemplation of the etiology of a case of an illness, a potential cause cannot be thought of merely in terms of the entity at issue. Critically important is also the timing of its occurrence. Given the inherently retrospective nature of the concept of etiology, and also the commonly more-or-less characteristic lag time from etiologic experience to the illness outcome, the timing of the etiologic experience is ordinarily to be considered on the scale of etiologic time defined by its zero point $\left(\mathrm{T}_{0}\right)$ at the time of the first manifestation(s) of the illness (as the time of actual onset remains unknown). It is on this scale that one can define etiologically meaningful "recent" exposure, for example, as a retrospective concept, one whose temporal referent is in negative etiologic time.

Any given etiologic entity has a time course over the entire range of etiologic time in which it could be etiologic to the illness at issue. This time course is not, in itself, a potential cause of the illness: the concept of cause is inherently comparative between two categories of a causal determinant; it involves a contrast of the causal, or index, category to a meaningful reference category, such as the contrast of a given degree of only recent exposure to no exposure at all, both in the entire potentially etiologic period. In this way, meaningful etiologic contrasts tend to be longitudinal in respect to etiologic time; and when interest in research focuses on a given segment of etiologic time, experiences in other segments of it tend to represent confounding by the very entity under study.

\section{The object of study}

Insofar as a given retrospective experience was causal to a case of illness, occurring at etiologic $\mathrm{T}_{0}$, it served to complete a sufficient cause for this outcome (time-specific) while the determinant's reference category would not have, all other component causes remaining the same.

Thus, the etiologic effect of a particular etiologic contrast means that the index history produces an increased incidence of the illness relative to the reference category - specifically, and notably, at etiologic $\mathrm{T}_{0}$. Since at issue is the occurrence of events at a particular moment in scientific time (etiologic $\mathrm{T}_{0}$ ), the concept of incidence here is not that of proportion-type incidence but incidence density (4). As people 'at risk' for the outcome event move over calendar time and age, they remain stationary at etiologic $\mathrm{T}_{0}$ and thus continually contribute to both the numerator and denominator inputs to the incidence densities (index and reference) at etiologic $\mathrm{T}_{0}$.

The object of an etiologic study thus generally is the outcome's incidence density in causal relation to the etiologic determinant in a defined domain. Commonly, several index categories are each contrasted with the same reference category; and for each contrast, the comparative parameter of principal interest is incidence-density ratio (4), for etiognostic purposes in particular $(3,5)$.

That the relation is of interest as a causal one must no longer be viewed as meaning that it meets whatever 'criteria' of causality, different from the outlook in the U.S. Surgeon General's epoch-making Smoking and Health (6) report for example. Rather, the modern imperative is to appreciate that the alternative to the relation's causality is confounding (7). In this framework the object can be viewed as the corresponding descriptive relation conditional on all potential confounders (i.e., extraneous determinants of the incidence rate) or, less restrictively yet sufficiently, on the values of a suitable scoring function based on them (8).

\section{The study population and study base}

Studying an etiologic occurrence relation (abstract) is, in all essence, tantamount to documenting its empirical counterpart in a study population's course over time (calendar time, say), to documenting experience of the study object's form, among representatives of the object's referent domain. The resulting empirical occurrence relation has the study population's follow-up, the aggregate 
of population-time resulting from this, as its referent, the empirical values of the comparative parameters having their direct meanings in reference to this population-time and this alone. For the purpose of inference about the object itself (with an abstract referent domain), however, this empirical referent constitutes but the basis of learning, the study base in this meaning of the aggregate of the person-moments contributing the denominator inputs to the documented rates.

Given the nature of the determinant categories in the object of an etiologic study, typically involving reference, in some sense, to 'recent' experience etc., the subpopulations that are constituted by representatives of these states of history (current at etiologic $\mathrm{T}_{0}$ ) within the study population have turnover of membership; they are 'dynamic' in this sense; they constitute what demographers term dynamic, or open (for exit), populations. As a consequence, the study population at large in an etiologic study typically is of this type, and not a cohort, a closed (for exit) population. (It bears note that our dictionary still does not define a dynamic population as one whose membership is defined by a state, for the duration of that state, and a cohort as one defined by an event, forever after; and it gratuitously posits the falsehood that "all natural populations are dynamic.")

The first step toward having a study population always is a commitment to a source population for it. When the study population is dynamic, it cannot be operationally 'formed' within some period of the source population's time course; it can only be defined within the source population. Central to this definition are, of course, representations, however fleetingly, of not only the study domain but also one of the contrasted histories of etiologic interest. As the study population remains inseparably imbedded in the source population, its requisite follow-up, forming the study base, is operationally a matter of following the source population and then narrowing the rate numerator and denominator inputs from this experience down to those that actually have to do with the study base itself. (Our dictionary still does not incorporate the term or concept of source population.)

In etiologic research there is an important duality of options in the definition of the source population. It can be given a direct, or primary, definition, such as the resident population of a given metropolitan area. The alternative is to give direct definition to the scheme by which cases of the illness are identified. This implies indirect, or secondary, definition of the source population - as the entirety of the population (dynamic) in the 'werewould' state of: were the illness now to occur, it would be identified by the defined scheme. The indirectly defined source population is the catchment population of the directly defined scheme of case identification. (Our dictionary defines catchment "area" only - as "Region from which clients of a particular health facility are drawn," gratuitously adding that it "may be well or ill defined.")

\section{Documentation of the occurrence relation}

The source population's follow-up is, first and foremost, a matter of identifying the cases that occur in this source population-time; and secondarily, their documentation has to do, apart from diagnosis, with the facts pertaining to the study domain and the etiologic history in the main. Narrowing this first-stage case series down to the cases that arose from the actual study population within the source population leads to the second-stage case series, to the study's ultimate rate numerator series, classified by the categories of the etiologic determinant.

The documentation of the sizes of the study population-times for each of the determinant categories involves a subtlety arising from the dynamic nature of the study population. Complete quantification of these segments of the study base, each involving an infinite number of person-moments, is impossible. The only option is sampling of the source population-time. This leads to the first-stage rate denominator series, and this is reduced to its corresponding second-stage series analogously with the numerator series.

These two series provide for the computation not of the empirical rates per se but their corresponding quasirates: if $c_{\mathrm{i}}$ cases in the study base arose from the $i$ th index population-time and $c_{0}$ from the reference population-time, and if the corresponding numbers in the ultimate denominator series are $d_{1}$ and $d_{0}$, then the corresponding quasi-rates are $c_{\mathrm{i}} / d_{\mathrm{i}}$ and $c_{0} / d_{0}$, and the corresponding empirical rate (incidence density) ratio is $\left(c_{\mathrm{i}} /\right.$ $\left.d_{\mathrm{i}}\right) /\left(c_{0} / d_{0}\right)$ - the actual, not quasi-, ratio (4). (Some authors (9) have recently invoked the term "pseudo-rates," even though there is nothing inauthentic or deceptive about them: they do not even have the inverse-time dimensionality of incidence density. Our dictionary does not recognize either term, nor any substitute for these.)

\section{Validity assurance}

A first-order requirement for the validity of an etiologic study is coherence between the numerator and denominator series: the former has meaning only insofar as it is either the entirety of cases occurring in a defined referent population-time or a fair sample of these, and the denominator series has meaning only insofar as it indeed is a fair sample of a defined population-time; and the pair generally has to have mutual coherence of their respective referents, both referring to the same population-time, to the study base. 
The assurance that this requirement gets to be met begins with case definition. When the source population is given a primary definition, the goal of complete case identification, or partial identification still independent of determinant histories, is well served by defining, for the purposes of the study, cases that are both severe and typical in their manifestations. And when the source population's definition is secondary to the scheme of case identification, such a restriction of the definition of cases serves to make the concept of the catchment population more concrete, thus facilitating its valid sampling, whether by the use of population rosters or by the use of cases of some extraneous outcomes (equally severe and typical). This was not yet understood in, say, the recent and eminent World Health Organization "case-control" study on oral contraceptive use in the etiology of pulmonary embolism (10), nor in a recent and eminent delineation of valid selection of "controls" in "case-control" studies (11).

Another principle in the pursuit of coherence for the two series involved in an etiologic study has to do with the definition of the source population for the study population, equally unappreciated at present. The operational aim in this is to see to it that case identification is a matter of canvassing all of the facilities of care for the illness at issue for a concretely defined and contiguous population such as the resident population of a metropolitan area.

When both of these elementary principles are heeded, the distinction between primary and secondary definition of the source population becomes moot, and this brings about an added advantage. Now the sampling for the (first-stage) denominator series involves two valid options so long as the pursuit of coherence is the concern: probability sampling by the use of an available population roster as a sampling frame, and the use of cases of extraneous outcomes obtained by canvassing the facilities that are relevant in their complete ascertainment. As for the latter option, representativeness of the referent of the numerator series requires also that the illnesses involved occur independently of the etiologic determinant at issue. And as this option now is available, it might be preferable to the statistical-type sampling on account of another requirement for validity, namely equi-accuracy of the determinant histories between the numerator and denominator series.

Beyond the topics of coherence and equi-accuracy of histories, the principal one among the others in the assurance of validity is that of the alternative to causality, that of preventing confounding in the results. Assurance of unconfoundedness of the results, just as the assurance of coherent referents, may have to begin in the operational definition of the elements in the object of study. Confounding is a feature of the study base before it possibly affects the results; and there it has to do with the distributions of the respective segments of the study base according to one or more extraneous determinants. When a confounder is not adequately documentable (for control in the analysis), the aim has to be the attainment of unconfounded contrasts on the level of the study base. In the 1980s, eminent studies addressed aspirin use in the etiology of Reye's syndrome in the domain of febrile illness in childhood, with great concern for confounding by level of fever which was not at all adequately documentable across the use vs. no use contrast (12). The solution would have been the operationalization of no aspirin use in terms of, say, acetaminophen use. In the study of occupational etiology of illness, it is commonplace to operationalize exposure and freedom from it in terms of particular plants or occupations, with concern for socioeconomic status as a potential confounder, again not subject to adequate documentation. And again, the solution is selectivity in these specifications with the aim of preventing confounding in the study base in the same sense as in the example above: by assuring balanced distributions without restricting the range of these.

It remains commonplace to hold and teach, as I also used to do, that one option in the prevention of confounding is restriction of the study domain and, hence, the study base to a narrow range of the potential confounder. Only later did I come to understand that this actually is a meaningful option in a particular kind if situation only. Consider studying the etiology of laryngeal cancer with focus on smoking in this and with alcohol use as a potential confounder, or vice versa. The potential confounder is, in its multitudinous potential patterns of qualitative and quantitative particulars over retrospective time, eminently resistant to very meaningful conceptualization even, to say nothing about meaningful documentation and control. But: the 'nonexposure' category is an exception to this, in this example and many others. Restriction to it provides for unconfounded results when attempted documentation and subsequent control are but half-measures to their respective ends.

\section{The fallacies sustaining matching}

The topic of prevention of confounding in etiologic research still routinely brings up that of matching; and I must plead guilty for this as much as anyone. Our dictionary defines the concept as "The process of making a study group and a comparison group comparable with respect to extraneous factors"; but it is mistaken. In an etiologic study, matching generally has to do with the selection of the first-stage denominator series, specifically selection in such a way that its distribution according to some codeterminant(s) becomes identical to that of the numerator series. These series are not 'comparison group" 
and 'study group,' respectively; and regardless, comparison and, hence, comparability of the two series is not an issue in an etiologic study, correctly understood.

Such is, still, the preoccupation with matching in etiologic research that Table 1 of the study report typically shows how nicely 'comparable' the 'control group' is with the 'case group' as a result of matching, even in respect to some unmatched codeterminants, the idea being that these potential confounders got to be prevented from becoming actual confounders and thus do not require control in the analysis. Yet, matching in the sampling of the study base has no bearing on confounding in the base itself; it does not serve to prevent confounding in it, and it thus does not justify not controlling it in analysis; and in any case, matching by a correlate of the determinant at issue necessitates accounting for this selectivity in the analysis even if there was no confounding in the study base (on account of this not being a potential confounder, a codeterminant).

Not only does matching in the sampling of the study base not serve to prevent confounding, but it always has the alternative of mere documentation and subsequent control based on this. It follows that it is never justifiable by validity pursuits, in sharp contrast to the ideas surrounding that 'Table 1' above.

Some seek to justify such matching by the pursuit of efficiency. Thinking about this issue is now held back by the ingrained notion that, in the selection of the denominator series ('control group'), one either matches on a given codeterminant or pays no attention to this codeterminant. So long as these remain the only options, it is indeed true that matched selection can be more efficient than indiscriminate selection. But once we get to appreciate that matching is but a special case of discriminate selection, it becomes apparent, for one, that efficient selections from the various confounder (or modifier) strata are proportional not to the respective numbers of cases per se but these divided by quantities that are proportional to the respective square roots of unit costs; and for another, the selection allocations need to be proportional to the square roots of the products of the proportions that the index and reference segments of the study population represent in the source population. With this understood, it becomes evident also that matching is, in all essence, never justifiable by efficiency pursuits, again in sharp contrast to prevailing beliefs.

\section{The cohort fallacy}

Our dictionary's definition of cohort study is so eminently confused that it does not deserve further attention here; but the dictionary gives "follow-up study" as a synonym for the term, and there the definition is rather clear: " $\mathrm{A}$ study in which individuals or populations selected on the basis of whether they have been exposed to risk ... are followed to assess the outcome of exposure ..."

Thus, a 'cohort study' documents prospective occurrence in relation to current determinant histories, both of these as of cohort, and scientific, $\mathrm{T}_{0}$, not the current occurrence at the time, $T_{0}$, as of which the histories are defined; and indeed, the synonyms of "cohort study" in the dictionary include "prospective study." In the framework of tenable concepts, however, interest in prospective occurrence is characteristic of prognostic studies, and not etiologic/etiognostic ones. The paradigm for causality-oriented prognostic studies is the randomized controlled trial, and it has been commonplace to hold this as a paradigm for etiologic studies as well. Yet, in that 'paradigm,' prospective occurrence is related to prospective divergence in the determinant, and this is not done in a 'cohort study' on etiology, much less in a true etiologic study. The need for a cohort-type study population is characteristic of prognostic studies, while etiologic/etiognostic ones generally require a dynamic study population, as discussed above.

When a cohort is followed for the purposes of etiologic research, it must be understood to be a source population, commonly for multiple and yet-to-be-specified etiologic studies. Typically, a wide range of health-related data are recorded in the course of the follow-up, prospectively. This does not constitute a study, only the development of a resource for studies. Any given etiologic study in this framework, when properly understood, is not one involving the 'cohort study' structure. Instead, it involves identification of the first-stage numerator series, sampling of the cohort population-time for the firststage denominator series, etc.; and, as a matter of particular note, the etiologic histories are not defined as a cohort $\mathrm{T}_{0}$ but as of the etiologic $\mathrm{T}_{0}$ - the person-moments of case occurrence and base probes. When, as is typical, the relevant histories change over the source cohort's follow-up, the study population within the cohort is dynamic.

\section{The trohoc fallacy}

According to our dictionary, trohoc study is "retrospective case-control study," while case-referent study, with "retrospective study" a synonym for the term, is one in which "the past history of exposure to a suspected risk factor is compared between "cases" and "controls"," the latter being "persons who resemble the cases in such respects as age and sex but do not have the disease or condition of interest." And further, the study may be "called 'retrospective' because it looks back from the outcome to is causes," in contrast to the prospective, 
causes-to-outcome orientation in a'cohort study.' It thus represents quite a complete reversal of the 'cohort' study concept, so that the 'trohoc' term is quite apposite - even if, according to our dictionary, its use is "deprecated by the great majority of epidemiologists."

Relative to the 'cohort' study, this 'trohoc' reversal of it has one great virtue: consonant with the inherent nature of etiologic objects of study, the determinant histories, such as 'recent' exposure, are defined as of the time of outcome, and not as of its antecedent cohort $T_{0}$.

From there on, alas, benightedness sets in. There is no understanding that, whereas an etiologic study generally is to address incidence in relation to etiologic determinants of this, follow-up of a study population of persons who have not yet experienced the outcome event at issue is inescapable. (Our dictionary still presents "follow-up study" as a synonym for "cohort study" but not for "trohoc-study" or "case-control study," as though the latter did not involve follow-up. And it defines "nested case-control study," as though some 'case-control' studies were not 'nested' in population follow-up.) If this were appreciated, then it would also be appreciated that the cases do not constitute a 'study group' but a rate numerator series; and from this would immediately flow the need, not for a 'control group' but, for a denominator series in the meaning of a fair sample of the study base, of the referent of the case series and, thus, of the study result. And given this understanding of the two 'groups,' quasi-rates specific to the contrasted histories would be understood to be the immediate results. Thus, concerns of comparability would focus on comparisons between these, while comparisons between the numerator and denominator series would be understood to be absurd, never providing for understanding, for example, the confounding alternative to causality.

\section{Last but not least: the objective}

Thus far, etiologic research has been motivated by the objective to find avenues for prevention, for community-level preventive interventions in particular. Indeed, our dictionary (2) defines epidemiology itself as "The study of the distribution and determinants of health-related states or events in specified populations, and the application of this study to control of health problems."

In the future, I believe, epidemiologic research will be seen as health-related occurrence research in human domains, conducted mainly within various health sciences rather than within 'epidemiology'; and as for its objectives, quintessentially applied epidemiologic research will dominate and be seen as serving to provide for the knowledge-base of the practice of health care, for dia-, etio- and prognosis in it, with effects of intervention addressed in prognosis-oriented research (2). In this framework, the knowledge-base of intervention-prognosis for prevention generally derives from etiologic research in a preliminary and incomplete way only, if at all, while etiognosis generally is dependent on etiologic research expressly directed to application in etiognosis.

With etiognosis a central concern in occupational medicine, as a matter of setting the probability that a given case of illness is occupational in its etiology (etiogenesis), it is important in occupational-etiologic research to appreciate what bearing this purpose has on study design. When provision for prevention is the purpose, the spirit of etiologic research is largely that of hypothesistesting; and even when estimation is the end, the etiologic histories and effect modifiers tend to be addressed in terms so superficial that quantification of "odds ratios" (incidence-density ratios) tends to be empty of meaning for etiognosis. Gnosis is a quantitative challenge, and it has to do with instances with quite specific gnostic profiles - occupational etiognosis with a particular variant of a 'positive' occupational history together with a particular set of realizations for known or suspected modifiers of the etiognostically relevant rate ratio. Failure to address the magnitude of the causal rate ratio as a function of these specifics in the object designs of etiologic studies has had the consequence that the already abundant etiologic research has but little bearing on etiognosis (3).

In the future, etiologic research even for etiognosis will be but a minor segment of epidemiologic research. Now that the advent of national health insurance has revolutionized the scope of public health concerns and the mantras in the latter have become quality assurance and cost-containment in clinical medicine in particular, epidemiologic research will increasingly serve the development of the knowledge-base of health care at large (3, $13,14)$, principally clinical diagnosis and prognosis in it, as well as the evaluation of the quality of health care in the context of whatever prevailing standards for it (14, 15).

\section{References}

1. Berlin I. The proper study of mankind. London: Chatto and Windus, 1997.

2. Last JM, editor. A dictionary of epidemiology. 3rd ed. New York (NY): Oxford University Press, 1995.

3. Miettinen OS. Evidence in medicine: invited commentary. Can Med Assoc J 1998;158:215-21.

4. Miettinen OS. Estimability and estimation in case-referent studies. Am J Epidemiol 1976;103:609-20.

5. Miettinen OS. Proportion of disease caused or prevented by a given exposure, trait or intervention. Am J Epidemiol 1974;99:325-32.

6. Smoking and health: report of the Advisory Committee 
to the Surgeon General of the Public Health Service. Washington (DC): US Government Printing Office, 1964. Public health service publication, no 1103.

7. Miettinen OS. Confounding and effect-modification. Am J Epidemiol 1974;100:350-3. Reprinted: ibid 1995;141: 1113-6.

8. Miettinen OS. Stratification by a multivariate confounder score. Am J Epidemiol 1976;104:609—20.

9. Rothman KJ, Greenland S. Modern epidemiology. 2nd ed. Philadelphia (PA): Lippincott-Raven Publishers, 1998.

10. World Health Organization Collaborative Study of Cardiovascular Disease and Steroid Hormone Contraception. Effect of different prostagens in low oestrogen oral contraceptives on venous thromboembolic disease. Lancet 1995;346:1582-
8.

11. Wacholder S, McLaughlin JK, Silverman DT, Mandel JS. Selection of controls in case-control studies, I: principles. Am $J$ Epidemiol 1992;135:1019-28.

12. Forsyth BW, Horwitz RI, Acampora D, Shapiro ED, Viscoli $\mathrm{CM}$, Feinstein AR, et al. New epidemiologic evidence confirming that bias does not explain the aspirin/Reye's syndrome association. JAMA 1989;261:2517-24.

13. Eisenberg J. Agency for Health Care Policy and Research. Ann Epidemiol 1998;8:283-5.

14. Miettinen OS. Up from Evidence-Based Medicine. Svepet. In press.

15. Epstein A. The outcomes movement - will it take us where we want to go? New Engl J Med 1990;323:266 —70. 